\title{
High levels of imported asymptomatic malaria but limited local transmission in KwaZulu-Natal, a South African malaria-endemic province nearing malaria elimination
}

Jaishree Raman ( $\nabla$ jaishreer@nicd.ac.za )

National Institute for Communicable Diseases https://orcid.org/0000-0003-0728-3093

\section{Laura Gast}

Clinton Health Access Initiative

Ryleen Balawanth

Clinton Health Access Initiative

Sofonias Tessema

University of California San Francisco

\section{Basil Brooke}

National Institute for Communicable Diseases

\section{Rajendra Maharaj}

South African Medical Research Council

\section{Givemore Munhenga}

National Institute for Communicable Diseases

\section{Power Tshikae}

National Institute for Communicable Diseases

\section{Vishan Lakan}

South African Medical Research Council

\section{Tshiama Mwamba}

National Institute for Communicable Diseases

\section{Hazel Makowa}

University of Pretoria

\section{Lindi Sangweni}

KwaZulu-Natal Provincal Malaria Control Programme

Moses Mkhabela

KwaZulu-Natal Provincial Malaria Control Programme

Nompumelelo Zondo

KwaZulu-Natal Provincial Malaria Control Programme

\section{Ernest Mohulatsi}

Humana People to People 


\section{Zuziwe Nyawo}

KwaZulu-Natal Provincial Malaria Control Programme

\section{Sifiso Ngxongo}

KwaZulu-Natal Provincial Malaria Control Programme

\section{Sipho Msimang}

KwaZulu-Natal Provincial Department of Health

\section{Nicole Dagata}

Clinton Health Access Initiative

\section{Bryan Greenhouse}

University of California San Francisco

\section{Lyn-Marie Birkholtz}

University of Pretoria

\section{George Shirreff}

Clinton Health Access Initiative

\section{Rebecca Graffy}

Clinton Health Access Initiative

\section{Bheki Qwabe}

KwaZulu-Natal Provincial Malaria Control Programme

\section{Devanand Moonasar}

South African National Department of Health

\section{Research}

Keywords: Malaria, South Africa, KwaZulu-Natal, residual transmission, asymptomatic carriage, elimination, vector control, rapid diagnostic tests, malaria importation, KAP

Posted Date: April 8th, 2020

DOI: https://doi.org/10.21203/rs.2.23648/v3

License: (c) (1) This work is licensed under a Creative Commons Attribution 4.0 International License. Read Full License

Version of Record: A version of this preprint was published at Malaria Journal on April 15th, 2020. See the published version at https://doi.org/10.1186/s12936-020-03227-3. 


\section{Abstract}

Background KwaZulu-Natal, one of South Africa's three malaria endemic provinces, is nearing malaria elimination, reporting fewer than 100 locally-acquired cases annually since 2010. Despite sustained implementation of essential interventions, including annual indoor residual spraying, prompt case detection using malaria rapid diagnostics tests and treatment with effective artemisinin-based combination therapy, low-level focal transmission persists in the province. This malaria prevalence and entomological survey was therefore undertaken to identify the drivers of this residual transmission.

Methods Malaria prevalence as well as malaria knowledge, attitudes and practices among community members and mobile migrant populations within uMkhanyakude district, KwaZulu-Natal were assessed during a community-based malaria prevalence survey. All consenting participants were tested for malaria by both conventional and highly-sensitive falciparum-specific rapid diagnostic tests. Finger-prick filterpaper blood spots were also collected from all participants for downstream parasite genotyping analysis. Entomological investigations were conducted around the surveyed households, with potential breeding sites geolocated and larvae collected for species identification and insecticide susceptibility testing. A random selection of households were assessed for indoor residual spray quality by cone bioassay.

Results A low malaria prevalence was confirmed in the study area, with only $2 \%(67 / 2979)$ of the participants found to be malaria positive by both conventional and highly-sensitive falciparum-specific rapid diagnostic tests. Malaria prevalence however differed markedly between the border market and community $(p<0001)$, with the majority of the detected malaria carriers $(65 / 67)$ identified as asymptomatic Mozambican nationals transiting through the informal border market from Mozambique to economic hubs within South Africa. Genomic analysis of the malaria isolates revealed a high degree of heterozygosity and limited genetic relatedness between the isolates supporting the hypothesis of limited local malaria transmission within the province. New potential vector breeding sites, potential vector populations with reduced insecticide susceptibility and areas with sub-optimal vector intervention coverage were identified during the entomological investigation.

Conclusion If KwaZulu-Natal is to successfully halt local malaria transmission and prevent the reintroduction of malaria, greater efforts needs to be placed on detecting and treating malaria carriers at both formal and informal border crossings with transmission blocking anti-malarials, while ensuring optimal coverage of vector control interventions is achieved.

\section{Background}

With a sustained national malaria incidence of less than 1 malaria case per 1000 population at risk, South Africa began implementing its 5-year malaria elimination strategy [1] in 2012. Achieving the country's elimination target of 2018 proved challenging due to financial and logistical constraints, resulting in suboptimal coverage of key interventions and an upsurge in cases [2]. Using the World Health Organization's Global Technical Strategy for Malaria 2016-2030 [3] as a guide, South Africa revised its 
malaria elimination strategy to include a phased district-level approach to malaria elimination [4]. As part of this phased approach, all three malaria-endemic districts within KwaZulu-Natal (KZN; Fig. 1a), one of South Africa's three malaria-endemic provinces, are being targeted for sub-national malaria elimination verification by 2021 .

KwaZulu-Natal bore the brunt of the 1999/2000 malaria epidemic, reporting in excess of 40000 cases and over 300 deaths in 2000 [5]. Drivers of the outbreak included the establishment of sulfadoxinepyrimethamine resistant Plasmodium falciparum parasites [6,7] and the emergence of pyrethroidresistant Anopheles funestus vector populations [8]. Responses to the outbreak included the successful implementation of an artemisinin-based combination therapy treatment policy, a first in Africa [9], and the re-introduction of dichlorodiphenyltrichloroethane (DDT)-based indoor residual spraying [10] in the province. Sustained implementation of these and other strategic interventions, including routine active case detection and a robust cross-border malaria collaboration with southern Mozambique and Eswatini [11], have substantially reduced reported case numbers and local transmission in KZN.

Since 2005, KZN has accounted for less than one percent of South Africa's malaria burden, with its three endemic districts, uMkhanyakude, King Cetshwayo and Zululand (Fig. 1b), achieving and maintaining very low transmission intensity status [12] since 2010 [5]. This, despite the regional upsurges in malaria cases in 2014 [13], and most recently in 2017 [2], when South Africa reported over 30000 cases, the highest number of cases since the 1999/2000 epidemic. While the current control strategies appear to provide adequate protection against upsurges, they have not stemmed persistent residual local transmission, particularly in uMkhanyakude district, KZN.

It is against this backdrop that this study was undertaken to identify, describe and define the factors driving and sustaining low-level malaria transmission in KZN. It is envisaged that the data generated will contribute to a holistic understanding of the various elements contributing to residual transmission in northern KZN, and inform elimination intervention selection and implementation toward sub-national malaria elimination by 2021 .

\section{Methods}

\section{Study site}

This study was conducted in areas identified as hotspots of local transmission, namely the municipalities of Jozini (a peri-urban area) and uMhlabuyalingana (rural, border area) in uMkhanyakude district, KZN, over a six-week period in February and March 2018. Malaria transmission in this region occurs mainly during the hot, wet summer months from September to May, with P. falciparum and Anopheles arabiensis 
the dominant parasite [14] and vector [15] species, respectively. Annual insecticide-based indoor residual spraying (IRS) of households in communities with reported locally-acquired cases is the core vector control intervention in this area. Spray operations generally take place at the beginning of the malaria season, between September and November. Standard malaria case management interventions included diagnosis using the First Response ${ }^{\circledR}$ falciparum-specific RDT (First Response ${ }^{\text {TM }}$ Malaria Ag P. falciparum HRP2 Detection Rapid Card Test, Premier Medical Corporation Ltd, India) or blood smears and treatment with the artemisinin-based combination therapy, artemether-lumefantrine (Coartem ${ }^{\circledR}$, Novartis Pharma, South Africa).

\section{Study design}

A mixed-methods approach was employed to facilitate an in-depth examination of potential malaria risk factors. The first approach was a community-based, household-level malaria prevalence survey in which participants were tested for malaria using standard and highly-sensitive falciparum-specific rapid diagnostic tests (RDTs), and were assessed on their malaria-related knowledge, attitudes and practices (KAP). To understand the movements of the migrant and mobile populations (MMPs) and their potential contribution to sustained transmission, the second component comprised an assessment of individuals visiting the KwaPhuza border market (Fig. 1c), situated along the border between uMhlabuyalingana municipality, KZN and Maputo Province, Mozambique, for malaria and determining their recent and typical travel history. The third component was an entomological survey of potential vector populations within the study area in order to assess malaria risk and receptivity.

\section{Sampling frame}

The community-based, household-level survey was restricted to two types of localities: those reporting at least one locally-acquired case (defined as a malaria infection acquired within that community as no travel to another malaria endemic region in past 14 days was reported) during the previous two malaria seasons, and those where MMPs are thought to frequent. As the edges of these communities are somewhat diffuse, the official administrative boundaries from Statistics South Africa (StatsSA) may potentially exclude portions of communities, so each StatsSA boundary was extended outwards by $1 \mathrm{~km}$. These extended borders defined the sample frame and study localities. To obtain the study sample, study localities were subdivided into a grid comprised of 500 X 500-meter blocks (Fig. 1c), and blocks were chosen at random (but with a probability proportion to the number of households therein). Inhabited blocks were randomly selected until 1,351 households were identified, with the caveat that more blocks were selected from within uMhlabuyalingana municipality, given its larger size, and the large number of localities that the MMPs were thought to frequent. Assuming every house in the block would be sampled, an average of 3.7 individuals per household (as calculated by the National Census) and an anticipated refusal or absence rate of approximately $20 \%$, sampling this number of households was designed to 
provide us with 4,000 study participants. Based on the study sample size calculation, if no individual was found to be malaria positive, this number of participants would be sufficient evidence to show a prevalence of less than $0.15 \%$.

At the KwaPhuza border market, a convenience sampling strategy was employed as the number of individuals using the border crossing is unknown and therefore unpredictable. All individuals entering the South African side of the border market on the day of sampling (every Wednesday during the 6-week survey period) were invited to participate. All those who consented were surveyed.

Entomological investigations were conducted in and within a $2 \mathrm{~km}$ radius of any surveyed household where a malaria case was detected during the survey. In study localities where no cases were detected, at least two randomly selected surveyed households were subjected to entomological investigations.

\section{Sample and field data collection}

\section{Blood and participant information collection}

All individuals over 2 years of age present at the selected household or visiting KwaPhuza border market were invited to participate in the study. Prior to blood sampling and survey administration, written consent was obtained from individuals $\geq 18$ years of age, with written consent from a guardian/caregiver/parent of individuals between 2 and 18 years. Assent was obtained from children aged between 6 and 18 years.

All consenting participants from the community and border market were tested for malaria by standard RDT (First Response ${ }^{\text {TM }}$ Malaria Ag P. falciparum HRP2 Detection Rapid Card Test, Premier Medical Corporation Ltd, India) and highly sensitive RDT (Alere ${ }^{\mathrm{TM}}$ Malaria AG P.F. Ultra Sensitive, Abbott, USA). This was done to compare the performance of the highly sensitive RDT to the standard RDT in a lowtransmission rural South African setting. Filter-paper finger-prick blood samples were collected on Munktell TFN cards (Munktell, Germany) and labelled with unique patient identifiers to ensure linkage of molecular and demographic data. Air-dried blood samples were packaged individually in zip-lock packets containing desiccant and transported to the National Institute for Communicable Diseases (NICD) in Johannesburg for further analysis. Malaria RDT-positive individuals were treated on-site with artemetherlumefantrine in accordance with South African malaria treatment guidelines [14].

A paperless KAP survey designed to efficiently gather information deemed critical by the KZN Malaria Control Programme was administered to all consenting participants. Participants over the age of six were asked general knowledge questions while those 18 years and older were asked more in-depth attitude and practice questions. Detailed travel histories from all consenting individuals 18 years and older, visiting the border market, were obtained using a semi-structured paper-based questionnaire. 


\section{Entomological activities}

At least two randomly surveyed households in each locality were visually inspected for indoor-resting adult mosquitoes. Households that received IRS during the current malaria season were randomly assessed for insecticide residual efficacy using the standard WHO cone bioassays [16] against an insecticide-sensitive An. arabiensis laboratory strain. Final mortality counts were taken 24 hours postexposure. Description and coordinates of any potential breeding site within a $2 \mathrm{~km}$ radius of a survey household identified during the entomological survey were recorded. Larvae, if present, were collected and transported to the malaria programme insectary in Jozini for further analysis.

\section{Laboratory analyses}

\section{Malaria asexual and sexual parasite detection}

A modification of the pooling PCR method described by Hsiang et al [17] was employed to confirm malaria infection and parasite species identification. Briefly, DNA extracted from master pools containing samples from five participants (two $6 \mathrm{~mm}$ in diameter samples per participant) using the QIAamp DNA mini extraction kit (Qiagen, Germany) was subjected to a cytochrome $b$ nested PCR. Samples from any positive master pool were tested individually, followed by Alul enzyme restriction digestion for species determination. All falciparum-positive samples were assessed for the markers associated with artemisinin and lumefantrine tolerance [18-20] as well as genotyped using 26 neutral microsatellite markers [21]. All collected blood samples, irrespective of malaria status, were also assessed for gametocyte carriage using the reverse transcriptase PCR method of Mlambo et al. [22].

\section{Vector species identification and susceptibility testing}

Larvae, reared to adulthood in the Jozini insectary, were morphologically identified using the keys of Gillies and Meillion [23] and Gillies and Coetzee [24], with sub-sets of the adults subjected to insecticide susceptibility testing using WHO susceptibility test kits [25]. Final mortality counts were taken 24 hours post-exposure. Species identity was confirmed where necessary using the PCR methods of Koekemoer et al. [26] and Scott et al. [27] for An. funestus group and An. gambiae complex samples, respectively.

\section{Malaria case classification}


Local case: a malaria case within a malaria receptive area where local transmission cannot be disproved and there is no recent history of travel to another malaria endemic area

Imported case: a malaria case whose source of infection can be trace to an area outside of South Arica where the patient has recently travelled.

\section{Statistical and geospatial analyses}

Data cleaning and analyses were conducted using Tableau Prep and Desktop (Tableau Software, Seattle, WA, USA), RStudio (RStudio, Vienna, Austria) and Stata 15.0 (Stata Corp, College Station, Texas, USA). Odds ratios (OR) associated with malaria risk were generated using univariate analysis and variable logistic regression models which took into correlations at the locality level. Confidence limits were set at $95 \%$ with $p<0.05$ considered significant. Geospatial mapping and analysis was conducted using ArcGIS (Esri, Redlands, California. USA).

\section{Results}

Initially 2096 community members from 1214 households consented to malaria testing and KAP survey administration. However, two participants withdrew their consent after receiving their test results, resulting a final study sample of 2094 participants from 1214 households. Willingness to participate in the survey was extremely high, with only one household head denying the survey team permission to administer the survey in their household. Data on the number of households where no one was present at the time of the survey were unfortunately not collected. Anecdotal evidence however suggests that this number was low. At the border-market, all 885 individuals approached, consented to malaria testing, and provided both travel histories and a filter-paper blood sample. The targeted sample size of 4000 was not achieved due to fewer participants than expected present at the selected households at the time of survey administration together with a week-long disruption of survey activities due to localized flooding.

Malaria prevalence was low, with only 67 (2\%) of the 2979 sampled individuals testing positive for malaria by the standard falciparum-specific malaria RDT. The malaria burden however differed significantly between the community $(2 / 2094)$ and border market $(65 / 885 ; p<0.0001)$, with the border market accounting for $97 \%(65 / 67)$ of all cases detected. Based on travel history data, all cases detected during this study, both at the border market and in the community, were classified as imported from neighbouring Mozambique. Good quality genotyping data were available for 46 parasite isolates $(68,7 \%)$ and revealed that the parasite isolates were genetically diverse and complex with limited genetic relatedness (Fig. 2), suggesting frequent and random mixing of parasites, consistent with the characteristics of imported infections from high transmission areas. Pairwise genetic relatedness assessment identified a single pair of highly related infections (identity by state (IBS) >0.6, Fig. 2c), 
suggesting limited direct transmission between these Mozambican individuals. Although case notification data confirmed that these infections were detected on the same day at the KwaPhuza bordermarket, information on source location within Mozambique was not collected. No additional cases were detected by ultra-sensitive RDT or pooled PCR, with gametocyte carriage limited to the 67 individuals found to be malaria positive. All parasite isolates carried the lumefantrine-tolerance marker but none of the validated artemisinin resistance markers.

Within the community, adult females were over-represented in the sample $(70.5 \%, \mathrm{p}<0.0001)$. The unemployment rate was incredibly high $(80 \%, p<0.0001)$ in spite of just over $50 \%(1031 / 2002)$ of the sampled population having secondary education or higher (Table 1). Previous exposure to malaria was extremely low, with only $1.6 \%(32 / 1998)$ of the sampled individuals reporting having had malaria. Despite the limited exposure to malaria, the majority of the survey participants $(94 \%)$, had heard of malaria (OR 2.70; $\mathrm{Cl}$ 2.52-2.88; $\mathrm{p}<0.0001$ ), and knew that if left untreated malaria could be fatal (OR 4.38; $\mathrm{Cl}$ 3.97-4.78; $\mathrm{p}<0.0001)$. Headaches $(86 \%)$ and having chills or feeling cold $(77 \%)$ were the two symptoms most frequently associated with malaria. Surprisingly, only $61.3 \%$ of the individuals interviewed associated fever with malaria. Although over $90 \%$ of all homesteads visited had been exposed to IRS operations during the current malaria season (OR 2.39; $\mathrm{Cl} 2.23-2.54 ; \mathrm{p}<0.0001)$, localities within Jozini municipality were more likely to be sprayed than those in uMhlabuyalingana municipality (OR 1.57; $\mathrm{Cl}$ 1.07-02.32; $p=0.022)$. Bed net ownership was very limited with only $4 \%(85 / 2090)$ of the individuals interviewed reporting owning a bed net, Use of a bed net differed markedly between the study municipalities, with usage significantly higher in the rural municipality of uMhlabuyalingana (53.2\%) compared to peri-urban Jozini (39.1\%; Table 1). Additional survey data can be found in supplementary Table 1.

In contrast to the community-based survey, similar numbers of males and females were sampled at the KwaPhuza border market $(\mathrm{p}=0.591)$. Males tested at the border market were more likely to be infected with malaria compared to the females (OR 1.98; Cl: 1.26-2.70; $p<0.0001)$. The majority of the individuals who provided travel information indicated that they were planning on travelling to major South African cities (Fig. 3).

All potential Anopheles breeding sites identified during the survey were geolocated, classified according to breeding status and mapped (Fig. 4). While no adult mosquitoes were found at any of the survey sites, larvae belonging to the An. gambiae complex and An. funestus group were collected from a number of potential sites across the study area. Molecular analysis confirmed the majority to be An. arabiensis (Table 2), with members of the An. funestus group extremely rare. Although all vector species identified in the study were found to be susceptible to DDT, populations of An. arabiensis and Anopheles pretoriensis 
with reduced pyrethroid-susceptibility were detected (Table 3). Quality of IRS operations varied between the different localities (Table 4).

\section{Discussion}

Achieving WHO-verified malaria elimination status in all three endemic districts in KZN by 2021 is a core objective of South Africa's current malaria elimination strategy. This study provided additional evidence of marked progress towards this goal in KZN, by confirming limited indigenous (autochthonous) malaria transmission in the province. However, vigilance in malaria surveillance and response must remain a priority, as the attainment of malaria elimination is under threat from numerous factors, including malaria importation from neighbouring high-prevalence countries and insecticide resistance.

The study parasite isolates were found to be highly diverse and complex with limited levels of genetic relatedness, distinctive features of parasites from a high transmission area. These findings supported the classification of all detected cases ( $97 \%$ of which were detected during the border market survey at the informal KwaPhuza border market) as imported based on travel history data. The rapid development of both formal and informal global transport networks has increased human mobility and the speed infectious diseases spread [28]. Within southern Africa where malaria distribution is extremely heterogeneous and country borders porous, human population movement (HPM), predominately by road [28], links areas of differing malaria receptivity. Areas of low receptivity, where malaria transmission would not normally be sustained, can experience persistent transmission [29] due to a continually replenished parasite pool [30]. This constant introduction of parasites into receptive areas by HPM has been identified as a major contributory factor in the failure of previous elimination campaigns [31].

All the identified malaria carriers in this study were asymptomatic, a likely consequence of acquired immunity due to Mozambique's higher transmission intensity. The majority of these malaria carriers would have most likely evaded passive case detection, potentially seeding and sustaining secondary

(introduced) transmission in receptive areas of KZN, had they not been detected and treated at the informal border crossing. In addition, many of MMPs interviewed indicated that they were transiting to major non-endemic cities within South Africa, potentially placing an increased burden on the health systems in these cities, if they became symptomatic and required treatment. As this survey was conducted outside of the peak transmission periods, it is very likely that levels of both HPM and asymptomatic carriage have been significantly underestimated, notwithstanding the likelihood that population movements differs across the year. This, together with the study findings strongly emphasize the critical role the mobile border malaria surveillance units play in reducing the opportunities for these asymptomatic carriers to seed secondary transmission in KZN, particularly as entomological investigations confirmed the presence of numerous receptive areas within the province. The ability of the 
mobile surveillance units to prevent onward transmission has been further enhanced with the recent addition of the gametocidal drug, primaquine, to their case management toolkit.

Acknowledging the threat asymptomatic malaria importation poses to malaria elimination in southern Africa, the MOSASWA (Mozambique, South Africa, Swaziland/Eswatini) cross-border collaboration has implemented two strategies to address asymptomatic malaria importation both in source and sink areas [32]. The first aims to reduce transmission in southern Mozambique by strengthening human capacity to effectively malaria control and improving intervention coverage in Maputo, Gaza and Inhanbane Provinces while the second focusses on detecting and treating malaria infections before they reach receptive areas within KZN. However, more detailed intelligence on the demographics and movements of the MMPs is required to inform targeted active case detection activities. Once this information is sourced, the appropriate effective active case detection activities must be expanded and concentrated at known border crossings (formal and informal) and gathering points of MMPs across South Africa, to facilitate the prompt detection of malaria and improved malaria awareness.

The current point of care diagnostic in South Africa, a conventional falciparum-specific RDT, appeared to be sensitive enough to detect asymptomatic carriage as no additional malaria cases were detected by standard PCR or ultra-sensitive falciparum-specific RDTs. While similar results have been observed in lowtransmission areas of the Gambia [33], they differ from a study in Ethiopia [34] which found the same ultra-sensitive RDT used in this study to be more sensitive compared to conventional RDTs when detecting asymptomatic malaria. A possible reason for this difference could be sensitivity differences between the conventional RDTs used in both studies. It has been suggested that low-density infections, including submicroscopic infection, are very prevalent in very low-transmission settings $[34,35]$ and are capable of sustaining transmission [36]. Detection of these infections however depends on the molecular methods used and more critically sample volume [37]. Unfortunately, the collection and appropriate storage of high-volume blood samples required for these ultrasensitive techniques was not logistically feasible during the survey. Investigations into the prevalence of low-density infections and their contribution to sustained transmission in KZN should be considered.

The severe under-sampling of school-going children and employed adults, a consequence of the community-based survey being conducted on weekdays between the hours of $09 \mathrm{~h} 00$ and $15 \mathrm{~h} 00$ when these populations groups were not present, was a major limitation of this study. As these groups are often highly mobile, their exclusion could have biased the finding of very limited indigenous transmission within the community. However, given the limited number of indigenous cases reported from the communities within the study area during the previous two malaria seasons, this seems highly unlikely. 
Encouragingly, despite KZN's current extremely low malaria prevalence, awareness of malaria and its associated dangers was high within the community. It was however surprising to note that while most participants knew the common signs and symptoms of malaria, headaches and feeling cold were more frequently listed than fever. A similar trend was observed in an earlier study [38], highlighting the need for targeted health promotion around fever and malaria, particularly as fever is used as a prescreening tool for malaria testing at all South African public health facilities.

Sustained effective coverage of efficacious vector control inventions is fundamental to the success of any elimination strategy [39]. The suboptimal IRS coverage driven by an increase in western-style homesteads and substandard spray quality detected in certain localities are potential obstacles to malaria elimination in KZN. In areas where IRS operations are still feasible, they must be closely monitored and evaluated, ideally in real time, to ensure the $90 \%$ coverage target of good quality IRS, is achieved. Additionally other vector control interventions such as improving housing structures and/or larval source management should be considered. Currently in $\mathrm{KZN}$, larval source management is employed in an ad hoc uncoordinated manner. The KZN control programme should consider upscaling its use; guided by regularly updated breeding site maps and comprehensive data management, to control out-door resting vector populations associated with sustaining residual transmission in $\mathrm{KZN}[15,40]$. As vector populations with reduced susceptibility to pyrethroids were detected in this study and have been confirmed in neighbouring Mozambique [41], it is imperative that resistance susceptibility testing becomes a routine programmatic activity, with the data generated used to inform insecticide selection.

\section{Conclusion}

This study identified the continual introduction of malaria parasites into receptive areas through asymptomatic importation from neighbouring Mozambique as a potential significant driver of residual indigenous transmission in northern KZN. Routine active detection at known informal border crossings, the introduction of the transmission-blocking drug primaquine and the strengthening of cross-borders initiatives are a few of the elimination strategies implemented to address imported and introduced malaria. It should be noted that current research suggests that if these interventions are to have any impact on suppressing indigenous transmission, a coverage of at least $80 \%$ must be achieved and maintained for a significant length of time. In addition, the receptivity and vulnerability of many localities within KZN was confirmed, highlighting the need for sustained, optimum coverage of a package of targeted effective vector control interventions.

\section{List Of Abbreviations}

DDT dichlorodiphenyltrichloroethane 
IRS indoor residual spraying

KAP knowledge, attitudes and practices

KZN KwaZulu-Natal

NICD National Institute for Communicable Diseases

MMPs migrant and mobile populations

MOSASWA Mozambique, South Africa, Swaziland/Eswatini

PCR polymerase chain reaction

RDT rapid diagnostic test

\section{Declarations}

\section{Ethics approval and consent to participate}

Approval for this study was received from the KwaZulu-Natal Provincial Department of Health, the Health Ethics Review Committee of the University of Witwatersrand (M170869), and Advarra Research Compliance Solutions (Maryland, USA). The University of Pretoria holds ethical approval for the genotyping analysis from the University of Pretoria's Health Sciences Committee (406-2014).

All individuals over 2 years of age present at the selected household or visiting KwaPhuza border market were invited to participate in the study. Prior to blood sampling and survey administration, written consent was obtained from individuals $\geq 18$ years of age, with written consent from a guardian/caregiver/parent of individuals between 2 and 18 years. Assent was obtained from children aged between 6 and 18 years.

\section{Consent for publication}

Consent to publish the data presented in this paper was obtained from the South African National and KwaZulu-Natal Provincial Departments of Health and National Institute for Communicable Diseases.

\section{Availability of data and materials}

The datasets used and/or analysed during this study are available from the corresponding author on reasonable request. 


\section{Competing interests}

The authors declare that they have no competing interests.

\section{Funding}

Financial support was provided through a Bill and Melinda Grant, a Clinton Health Access Initiative grant, and NICD research funding to JR; Wits Research Institute for Malaria and NICD research funding to BB, GM and PT; a Global Fund Grant and South African Medical Research Council research funding to RM and VL; the South African Research Chairs Initiative of the Department of Science and Technology, administered through the South African National Research Foundation to LB.

\section{Authors' contributions}

JR conceived the study and designed the study and analysis with $L G, R B, B B, R M, G M, P T, V L, S M, R G, G S$, $\mathrm{ND}, \mathrm{BQ}$ and $\mathrm{DM}$. BB, RM, GM, PT, ZN and SN led the entomological field and laboratory investigations. JR, LG, RB, LS, MM, NZ and EM oversaw the administration of the KAP and prevalence survey, collection and shipment of field samples. TM and JR optimized and conducted certain parasite molecular assays while ST, BG, HM and LB optimized and conducted the parasite relatedness assays. JR and LG conducted the statistical analysis, while LG generated the spatial maps. JR drafted the manuscript and all authors reviewed the manuscript critically for critical intellectual content, read and approved the final version of the manuscript.

\section{Acknowledgements}

The authors wish to the thank all the study participants for their willingness to participant in the survey, the entire KZN malaria programme for all their support prior, during and after the survey, the Bill and Melinda Gates Foundation, the Global Fund to Fight AIDS, Tuberculosis and Malaria, the Clinton Health Access Initiative, the South African Medical Research Council, the South African National Institute for Communicable Diseases and the Universities of California-San Francisco, Pretoria and Witwatersrand for financial and/or logistic support and Prof John Frean for critically reviewing the draft manuscript.

\section{References}

1. South Africa National Department of Health. Malaria elimination strategy for South Africa 20122018. Pretoria: South African National Department of Health; 2012

2. South African Development Community. SADC Malaria Status Report, 2017. 
3. Global technical strategy for malaria 2016-2030. Geneva: World Health Organization; 2015. https://www.who.int/malaria/publications/atoz/9789241564991/en/ Accessed 03 Jan 2020

4. South Africa National Department of Health. Malaria elimination strategic plan for South Africa 2019-2023. Pretoria: South African National Department of Health; 2019

5. Raman J, Morris N, Frean J, Brooke B, Blumberg L, Kruger P, et al. Reviewing South Africa's malaria elimination strategy (2012-2018): progress, challenges and priorities. Malar J. 2016;15:438.

6. Bredenkamp BL, Sharp BL, Mtembu SD, Durrheim DN, Barnes KI. Failure of sulfadoxinepyrimethamine in treating Plasmodium falciparum malaria in KwaZulu-Natal. S Afr Med J. 2001;91:970-2.

7. Roper C, Pearce R, Bredenkamp B, Gumede J, Drakeley C, Mosha F, et al. Antifolate antimalarial resistance in southeast Africa: a population-based analysis. Lancet. 2003;316:1174-81.

8. Hargreaves K, Koekemoer LL, Brooke BD, Hunt RH, Mthembu J, Coetzee M. Anopheles funestus resistant to pyrethroid insecticide in South Africa. Med Vet Entomol. 2000;14:181-9.

9. Barnes KI, Durrheim DN, Little F, Jackson A, Mehta U, Allen E, et al. Effect of artemether-lumefantrine policy and improved vector control on malaria burden in KwaZulu-Natal, South. PLoS Med. 2005;11:e330.

10. Maharaj R, Mthembu DJ, Sharp BL. Impact of DDT re-introduction on malaria transmission in KwaZulu-Natal. S Afr Med J. 2005;95:871-4.

11. Maharaj R, Moonasar D, Baltazar C, Kunene S, Morris N. Sustaining control: lessons from the Lubombo spatial development initiative in southern Africa. Malar J. 2016;15:409

12. A framework for malaria elimination. Geneva: World Health Organization; 2017. https://www.who.int/malaria/publications/atoz/9789241511988/en/ Accessed 03 Jan 2020

13. South African Development Community, SADC Malaria Status by 2014 Report, 2015.

14. South African National Department of Health. National guidelines for the treatment of malaria, South Africa, 2018. Technical report, South African Department of Health, Pretoria, South Africa. 2018.

15. Dandalo LC, Brooke BD, Munhenga G, Lobb LN, Zikhali J, Ngxongo SP, et al. Population dynamics and Plasmodium falciparum (Haemosporida: Plasmodiidae) infectivity rates for the malaria vector Anopheles arabiensis (Diptera: Culicidae) at Mamfene, KwaZulu-Natal, South Africa. J Med Entomol. 2017;54:1758-66.

16. Test procedure for insecticide resistance monitoring in malaria vectors, bio-efficacy and persistence of insecticides on treated surfaces. Geneva, World Health Organization. 1998 1-46. https://apps.who.int/iris/bitstream/handle/10665/64879/WHO_CDS_CPC_MAL_98.12.pdf? sequence $=1$ Accessed 03 Jan 2020

17. Hsaing MS, Lin M, Dokomajilar C, Kemere J, Plicher CD, Dorsey G, et al. PCR-based pooling of dried blood spots for detection of malaria parasites: optimization and application to a cohort of Ugandan children. J Clin Microbiol 2010:48:3539-43. 
18. Talundzic E, Chenet SM, Goldman IF, Patel DS, Nelson JA, Plucinski MM, et al. Genetic analysis and species specific amplification of the artemisinin resistance-associated kelch propeller domain in falciparum and P. vivax. PLoS ONE. 2015;10:e0136099.

19. Price RN, Uhlemann AC, Brockman A, McGready R, Ashley E, Phaipun L, et al. Mefloquine resistance in Plasmodium falciparum and increased pfmdr1 gene copy number. Lancet. 2004;364:438-47.

20. Sutherland CJ, Haustein T, Gadalla N, Amstrong M, Doherty JF, Choidini PL. Chloroquine-resistant Plasmodium falciparum infections among UK travellers returning with malaria after chloroquine prophylaxis. Antimicrob Agents Chemother. 2007;59:1197-9.

21. Tessema S, Wesolowski A, Chen A, Murphy M, Wilheim J, Mupiri A-R, et al. Using parasite genetic and human mobility data to infer local and cross border malaria connectivity in Southern Africa. eLife. 2019;8:e43510.

22. Mlambo G, Vasquez Y, LeBlanc R, Sullivan D, Kumar N. Short report: A filter paper method for the detection of Plasmodium falciparum gametocytes by reverse transcription-polymerase chain reaction. Am J Trop Med Hyg. 2008;78:114-6.

23. Gillies MT, de Meillion BL. The Anophelinae of Africa south of the Sahara (Afrotropical Region). 1968, Johannesburg: South African Institute for Medical Research.

24. Gillies MT, Coetzee M. A supplement to the Anophelinae of Africa south of the Sahara (Afrotropical Region). 1987, Johannesburg: South African Institute for Medical Research

25. Test procedures for insecticide resistance monitoring in malaria vector mosquitoes. Geneva, World Health Organization, 2018. 1-54

https://apps.who.int/iris/bitstream/handle/10665/250677/9789241511575-eng.pdf? sequence=1 Accessed 03 Jan 2020

26. Koekemoer LL, Kamau L, Hunt RH, Coetzee M. A cocktail polymerase chain reaction assay to identify members of the Anopheles funestus (Diptera: Culicidae) group. Am J Trop Med Hyg. 2002;66:804-11.

27. Scott JA, Brogdon WG, Collins FH. Identification of single specimens of the Anopheles gambiae Am J Trop Med Hyg. 1993;49:520-9.

28. Strano E, Viana MP, Sorichetta A, Tatem AJ. Mapping road network communities for guiding disease surveillance and control strategies. Sci Rep. 2019;8:4744.

29. Silal SP, Little F, Barnes KI, White LJ, Predicting the impact of border control on malaria transmission: a simulated focal screen and treat campaign. Malar J. 2015;14:268.

30. Tatem AJ, Jai P, Ordanovich D, Falkerner M. Huang Z, Howes R, et al. The geography of imported malaria to non-endemic countries: a meta-analysis of nationally reported statistics. Lancet Infect Dis. 2017; 17:98-107.

31. Saita A, Pan-ngum W, Phuanukoonnon S, Sriwichai P, Silawan T, White LJ, et al. Human population movement and behavioural patterns in malaria hotspots on the Thai-Myanmar border: implications for malaria elimination. Malar J. 2019;18:64.

32. Moonasar D, Maharaj R, Kunene S, Candrinho B, Saute F, Ntshalintshali N, et al. Towards malaria elimination in the MOSASWA (Mozambique, South Africa and Swaziland) region. Malar J. 
2016;15:419.

33. Mwesigwa J, Slater H, Bradley J, Saidy B, Ceesay F, Wittaker C, et al. Field performance of the highly sensitive rapid diagnostic test in a setting of varying malaria transmission. Malar J. 2019;18:288

34. Girma S, Cheaveau J, Mohon AN, Marasignhe D, Legese R, Balasingam N, et al. Prevalence and epidemiological characteristics of asymptomatic malaria based on ultrasensitive diagnostics: a cross-sectional study. Clin Infect Dis. 2019;69:1003-10.

35. Awandu SS, Raman J, Bousema T, Birkholtz L-M. Ultralow-density Plasmodium falciparum infections in African settings. Clin Infect Dis. 2019. Epub ahead of print.

36. Chen I, Clarke SE, Gosling R, Hamainza B, Killeen G, Magill A, et al. Asymptomatic malaria: a chronic and debilitating infection that should be treated. PLoS Med. 2016;19:e1001942.

37. Imwong M, Nguyen TN, Tripura R, Peto TJ, Lee SJ, Lwin KM, et al. The epidemiology of subclinical malaria infections in South-East Asia: findings from cross-sectional surveys in Thailand-Myanmar border areas, Cambodia and Vietnam. Malar J. 2015;14:381.

38. Manana PN, Kuonza L, Musekiwa A, Mpangane HD, Koekemoer LL. Knowledge, attitudes and practices on malaria transmission in Mamfene, KwaZulu-Natal Province, South Africa 2015. BMC Public Health. 2018;18:41.

39. Stuckey EM, Miller JM, Littrell M, Chitnis N, Steketee R. Operational strategies of anti-malarial drug campaigns for malaria elimination in Zambia's southern province: a simulation study. Malar J. 2016;15:148.

40. Burke A, Dandalo L, Munhenga G, Dahan-Moss Y, Mbokazi F, Ngxongo S, et al. A new malaria vector mosquito in South Africa. Sci Rep. 2017;7:43779.

41. Riveron JM, Huijben S, Tchapga W, Tchouakui M, Wondji MM, Tchoupo M, et al. Escalation of pyrethroid resistance in the malaria vector Anopheles funestus induces a loss of efficacy of PBObased insecticide-treated nets in Mozambique. J Infect Dis. 2019

\section{Tables}

Table 1. Demographic characteristics and risk factors associated with Plasmodium falciparum malaria from the community-based KAP survey by study municipality in uMkhanyakude district, KwaZulu-Natal 


\begin{tabular}{|c|c|c|c|c|c|c|}
\hline Risk Factor & $\begin{array}{l}\text { Jozini } \\
\mathrm{N}(\%)\end{array}$ & $P$ value & $\begin{array}{l}\text { uMhlabuyalingana } \\
\text { N (\%) }\end{array}$ & $\mathrm{P}$ value & $\begin{array}{l}\text { Total } \\
\text { N (\%) }\end{array}$ & $P$ value \\
\hline \multicolumn{7}{|l|}{ Gender } \\
\hline Female & $368(64.7)$ & $<0.0001$ & $1109(72.7)$ & $<0.0001$ & 1477 (70.5) & $<0.0001$ \\
\hline Male & $201(35.3)$ & & $416(27.3)$ & & 617 (29.5) & \\
\hline \multicolumn{7}{|l|}{ Age (year) } \\
\hline Under 5 & $43(7.4)$ & - & $35(2.3)$ & - & $77(3.7)$ & - \\
\hline $5-20$ & $71(12.5)$ & $<0.0001$ & $141(9.3)$ & $<0.0001$ & $212(10.1)$ & $<0.0001$ \\
\hline $21-60$ & $386(67.8)$ & $<0.0001$ & $1084(71.1)$ & $<0.0001$ & $1470(70.2)$ & $<0.0001$ \\
\hline Over 60 & $70(12.3)$ & $<0.0001$ & 265 (17.4) & $<0.0001$ & 335 (16) & $<0.0001$ \\
\hline \multicolumn{7}{|l|}{ Education } \\
\hline No Education & $131(23)$ & - & $403(26.4)$ & - & $534(25.5)$ & - \\
\hline Primary Education & $53(9.3)$ & $<0.0001$ & $264(17.3)$ & $<0.0001$ & $317(15.1)$ & $<0.0001$ \\
\hline \multicolumn{7}{|l|}{ Secondary } \\
\hline Education and Above & $252(44.3)$ & $<0.0001$ & $657(43.8)$ & $<0.0001$ & $909(43.1)$ & $<0.0001$ \\
\hline \multicolumn{7}{|l|}{ Employed } \\
\hline \multicolumn{7}{|l|}{ Yes } \\
\hline No & $172(30.1)$ & $<0.0001$ & $282(18.5)$ & $<0.0001$ & $454(21.7)$ & $<0.0001$ \\
\hline Previously had malaria & 399 (69.9) & & 1241 (81.5) & & $1640(78.3)$ & \\
\hline \multicolumn{7}{|l|}{ Yes } \\
\hline \multicolumn{7}{|l|}{ No } \\
\hline IRS in the past 6 months & $7(1.4)$ & $<0.0001$ & $25(1.7)$ & $<0.0001$ & $32(1.6)$ & $<0.0001$ \\
\hline Yes & 509 (98.6) & & $1457(98.3)$ & & 1966 (98.4) & \\
\hline \multicolumn{7}{|l|}{ No } \\
\hline \multicolumn{7}{|l|}{ Bed net ownership } \\
\hline Yes & $524(93.9)$ & $<0.0001$ & $1383(90.8)$ & $<0.0001$ & 1907 (91.6) & $<0.0001$ \\
\hline No & $34(6.1)$ & & $141(9.2)$ & & $175(8.4)$ & \\
\hline \multicolumn{7}{|l|}{ Bed net use } \\
\hline \multicolumn{7}{|l|}{ Yes } \\
\hline No & $23(4)$ & $<0.0001$ & $62(4)$ & $<0.0001$ & $85(4)$ & $<0.0001$ \\
\hline
\end{tabular}


Yes

No

\begin{tabular}{|c|c|c|c|c|c|}
\hline 9 (39.1) & $<0.0001$ & $33(53.2)$ & $<0.0001$ & $42(49.4)$ & $<0.0001$ \\
\hline 14 (60.9) & & $29(46.8)$ & & $43(50.6)$ & \\
\hline $20(3.9)$ & $<0.0001$ & $62(4.2)$ & $<0.0001$ & $82(4.1)$ & $<0.0001$ \\
\hline 497 (96.1) & & 1421 (95.8) & & 1918 (95.9) & \\
\hline
\end{tabular}

Table 2. PCR-based species identification of a subset of Anopheles larvae collected from 10 potential breeding sites within the study localities in uMkhanyakude district, KwaZulu-Natal, during the malaria prevalence survey in 2018

\begin{tabular}{llll}
\hline Complex/group & Total N & Species & Total N (\%) \\
\hline An. gambiae complex & 80 & An. arabiensis & $56(70)$ \\
& & An. quadriannulatus & $21(26)$ \\
& & Not identified & $3(4)$ \\
An. funestus group & 2 & An. parensis & $1(50)$ \\
& & Not identified & $1(50)$ \\
\hline
\end{tabular}

Table 3. Insecticide susceptibility status of Anopheles mosquito samples ${ }^{\text {a }}$ collected from potential breeding sites within uMkhanyakude district, KwaZulu-Natal, during the prevalence survey in 2018. 


\begin{tabular}{lllll}
\hline Species & Insecticide & $\begin{array}{l}\text { Number of mosquitoes } \\
\text { tested }\end{array}$ & $\begin{array}{l}\text { \% mortality 24hrs post } \\
\text { exposure }\end{array}$ & $\begin{array}{c}\text { Susceptibility } \\
\text { status }\end{array}$ \\
\hline An. & & 20 & 100 & Susceptible \\
gambiae complex & $4 \% \mathrm{DDT}$ & 11 & 100 & Susceptible \\
An. pretoriensis & $4 \% \mathrm{DDT}$ & 5 & 80 & Resistant \\
An. arabiensis & $0.05 \%$ & & 85.7 & Resistant \\
& Deltamethrin & 7 & & \\
An. pretoriensis & 0.05\% & & & \\
& Deltamethrin & & & \\
\end{tabular}

${ }^{\mathrm{a}}$ Larvae of mixed gender were reared to adulthood (2-3 days old) in the Jozini insectary and subjected to the standard WHO insecticide susceptibility testing assay

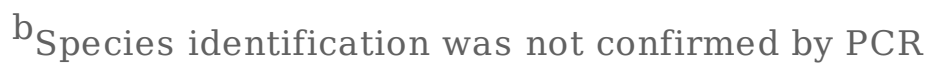

Table 4. Assessment of insecticide residual efficacy in selected households from study localities that had IRS in the past 6 months using cone bioassays with insecticide-sensitive laboratory-bred Anopheles arabiensis mosquitoes 


\begin{tabular}{|c|c|c|c|c|c|c|c|}
\hline \multirow[t]{4}{*}{ Municipality } & \multirow[t]{4}{*}{ Locality } & \multirow{4}{*}{$\begin{array}{l}\text { Date of last IRS } \\
\text { Round }\end{array}$} & \multirow{4}{*}{$\begin{array}{l}\text { Surface } \\
\text { Type }\end{array}$} & \multirow{4}{*}{$\begin{array}{l}\text { Insecticide } \\
\text { Used }\end{array}$} & \multicolumn{3}{|c|}{ \% Mortality after 24} \\
\hline & & & & & hours* & & \\
\hline & & & & & Top\# & & Middle $^{\#}$ \\
\hline & & & & & \multicolumn{2}{|c|}{ Bottom\# } & \\
\hline uMhlabuyalingana & Makanis & 2017 Oct 19 & Cement & DDT & 100 & 100 & 60 \\
\hline uMhlabuyalingana & Makanis & 2017 Oct 19 & Painted & DDT & 100 & 100 & 100 \\
\hline uMhlabuyalingana & Manguzi & 2017 Oct 19 & Painted & Deltamethrin & 100 & 90 & 90 \\
\hline uMhlabuyalingana & Manguzi & 2017 Oct 19 & Painted & Deltamethrin & 100 & 80 & 100 \\
\hline uMhlabuyalingana & Manguzi & 2017 Oct 18 & Painted & Deltamethrin & 100 & 100 & 100 \\
\hline uMhlabuyalingana & Mshudu & 2017 Nov 1 & Cement & DDT & 100 & 100 & 90 \\
\hline uMhlabuyalingana & Muzi & 2017 Dec 5 & Painted & Deltamethrin & 100 & 70 & 100 \\
\hline uMhlabuyalingana & Muzi & 2017 Dec 5 & Cement & DDT & 100 & 100 & 80 \\
\hline uMhlabuyalingana & Muzi & 2018 Jan 15 & Painted & DDT & 100 & 100 & 100 \\
\hline uMhlabuyalingana & Muzi & 2018 Jan 15 & Painted & Deltamethrin & 100 & 100 & 100 \\
\hline uMhlabuyalingana & Muzi & 2018 Jan 15 & Painted & Deltamethrin & 100 & 100 & 90 \\
\hline Jozini & Ndumo & 2017 Oct 4 & Mud & DDT & 100 & 100 & 100 \\
\hline Jozini & Ndumo & 2017 Oct 4 & Cement & DDT & 100 & 100 & 100 \\
\hline Jozini & Ndumo & 2017 Oct 4 & Painted & Deltamethrin & 100 & 100 & 100 \\
\hline Jozini & Ndumo & 2017 Oct 4 & Cement & DDT & 100 & 100 & 100 \\
\hline Jozini & Ndumo & 2017 Nov 14 & Painted & DDT & 93 & 100 & 94 \\
\hline Jozini & Ndumo & 2017 Nov 14 & Cement & DDT & 100 & 100 & 100 \\
\hline Jozini & Ndumo & 2017 Dec 20 & Painted & Deltamethrin & 100 & 100 & 100 \\
\hline Jozini & Ndumo & 2017 Dec 20 & Plastered & DDT & 100 & 100 & 92 \\
\hline Jozini & Ndumo & 2017 Dec 21 & Painted & Deltamethrin & 100 & 100 & 85 \\
\hline Jozini & Ndumo & 2017 Dec 20 & Cement & DDT & 100 & 100 & 100 \\
\hline uMhlabuyalingana & Ngutshana & 2017 Jan 20 & Painted & Deltamethrin & 60 & 50 & 90 \\
\hline Jozini & Shemula & 2018 Jan 30 & Painted & Deltamethrin & 100 & 100 & 100 \\
\hline uMhlabuyalingana & Sihangwani & 2017 Sep 20 & Plastered & DDT & 60 & 40 & 90 \\
\hline uMhlabuyalingana & Sihangwani & 2017 Sep 20 & Painted & Deltamethrin & 100 & 70 & 60 \\
\hline uMhlabuyalingana & Sihangwani & 2017 Sep 20 & Plastered & DDT & 100 & 90 & 100 \\
\hline uMhlabuyalingana & Tetepan & 2017 Nov 3 & Cement & Deltamethrin & 100 & 100 & 100 \\
\hline uMhlabuyalingana & Tetepan & 2017 Nov 3 & Cement & Deltamethrin & 90 & 100 & 100 \\
\hline
\end{tabular}




\begin{tabular}{llllllll} 
uMhlabuyalingana & Tetepan & 2017 Nov 3 & Cement & Deltamethrin & 100 & 100 & 100 \\
uMhlabuyalingana & Tetepan & 2017 Nov 3 & Cement & Deltamethrin & 100 & 100 & 100 \\
\hline
\end{tabular}

*A control was included in each experiment, with 100\% survival reported

\# Cones were place at three points on the wall surface of each selected homestead, the top, middle and bottom, to assess spray-quality across the entire wall surface

\section{Figures}

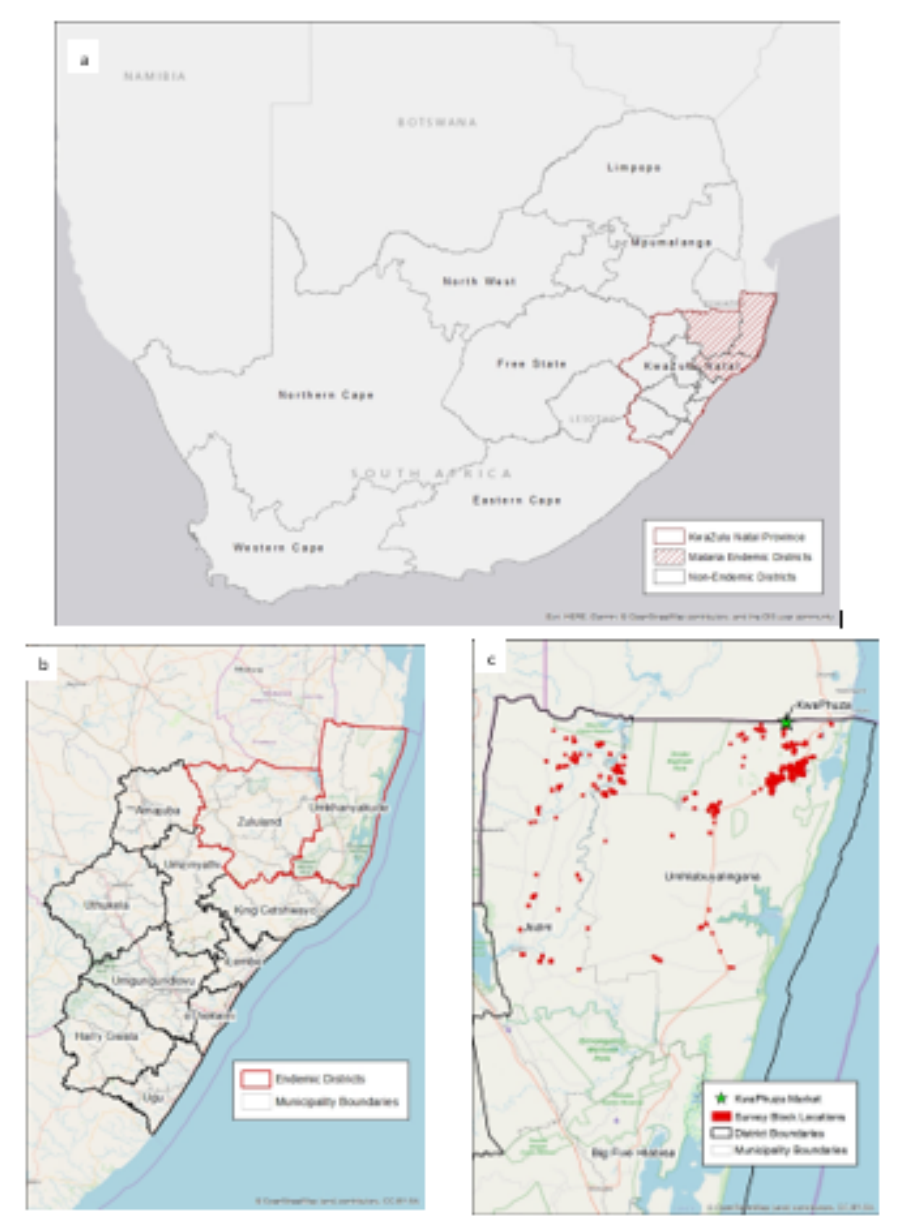

\section{Figure 1}

a) Malaria endemic and non-endemic provinces in South Africa; b) the endemic and non-endemic districts of KwaZulu-Natal and c) the localities sampled within the community and by the mobile surveillance units. Black arrow denotes KwaPhuza border market. 
A.

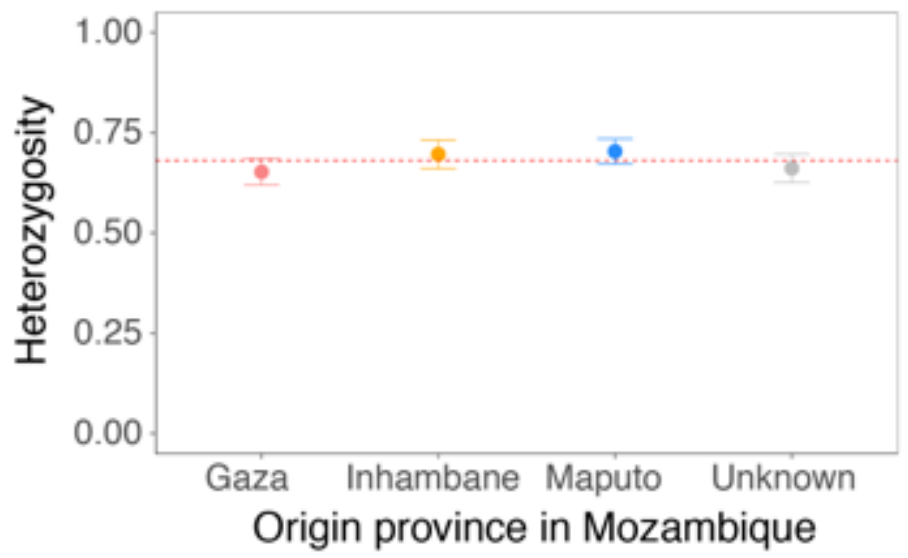

B.

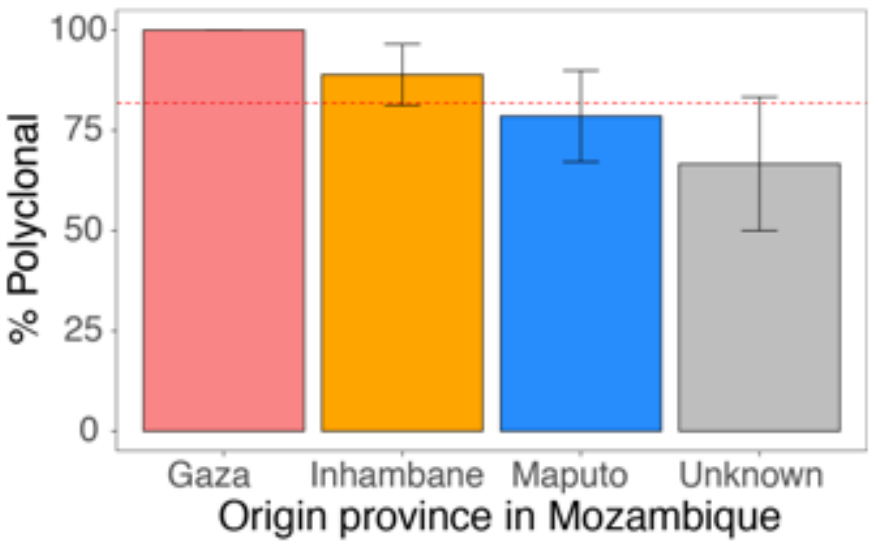

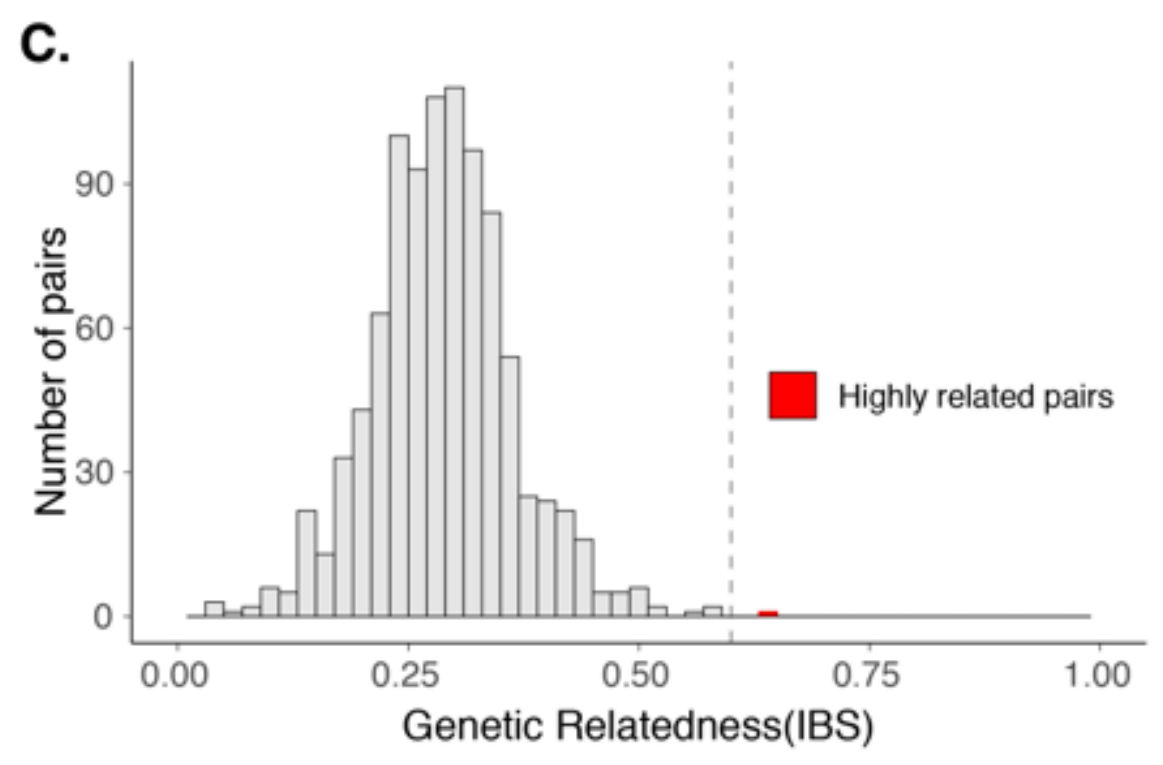

Figure 2

Within-host and population diversity of malaria parasite isolates collected during the prevalence survey, a) population level genetic diversity measured as the distribution of heterozygosity in 26 microsatellites. The dashed red line indicates the mean heterozygosity $=0.68$. Values for He range from $0-1$, with 0 representing no diversity and 1 representing $100 \%$ of alleles being different. b) complexity of the infections as measured by number of clones present by source locality with Mozambique and c) pairwise genetic relatedness between samples, calculated using identity by state (IBS) metric including all alleles detected in polyclonal samples. A single highly related pair (genetic relatedness $>0.6$ ) was identified and is shown in red. 

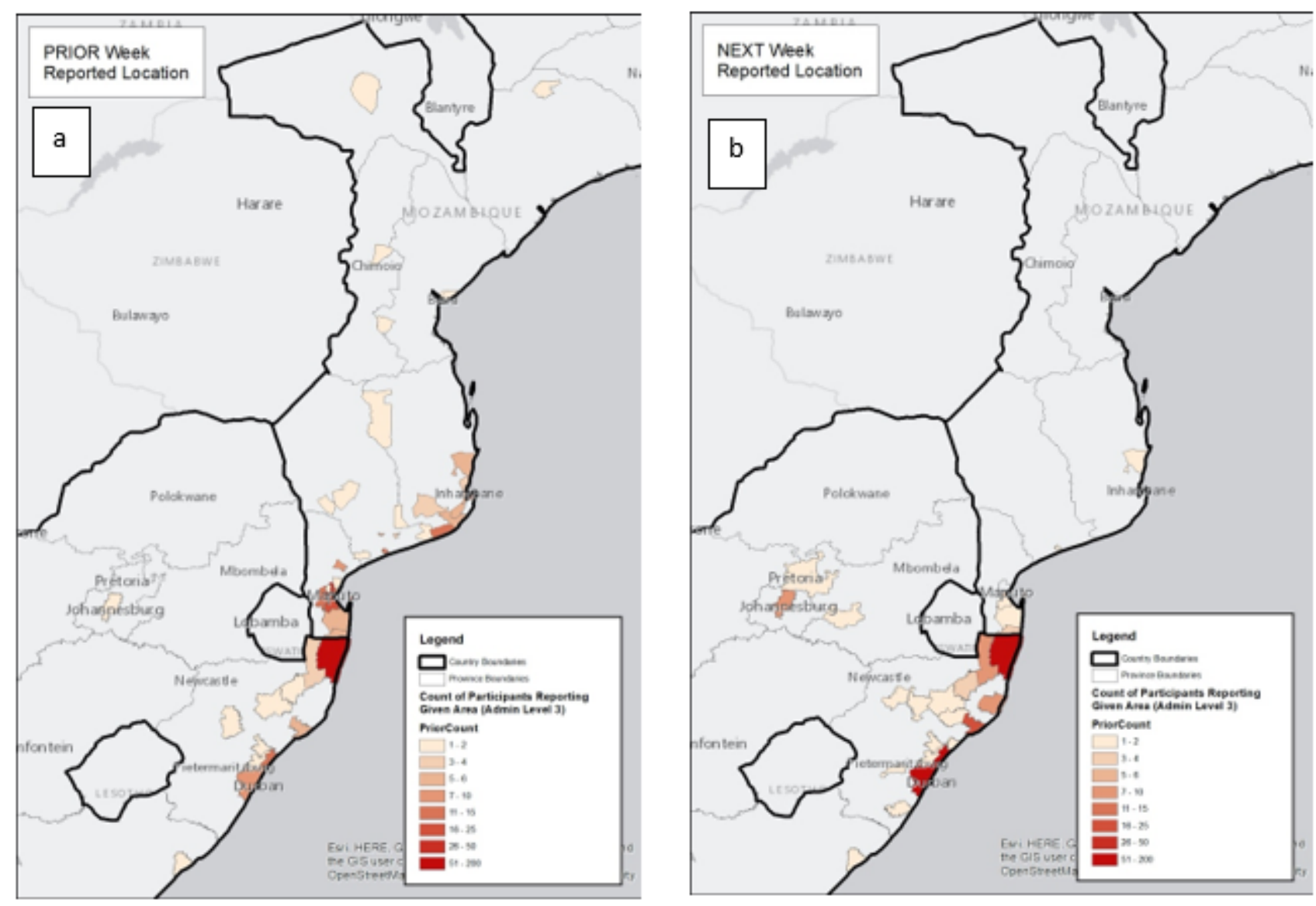

Figure 3

a) Localities the individuals sampled at KwaPhuza Border Market arrived from and b) localities to which they were transiting. 

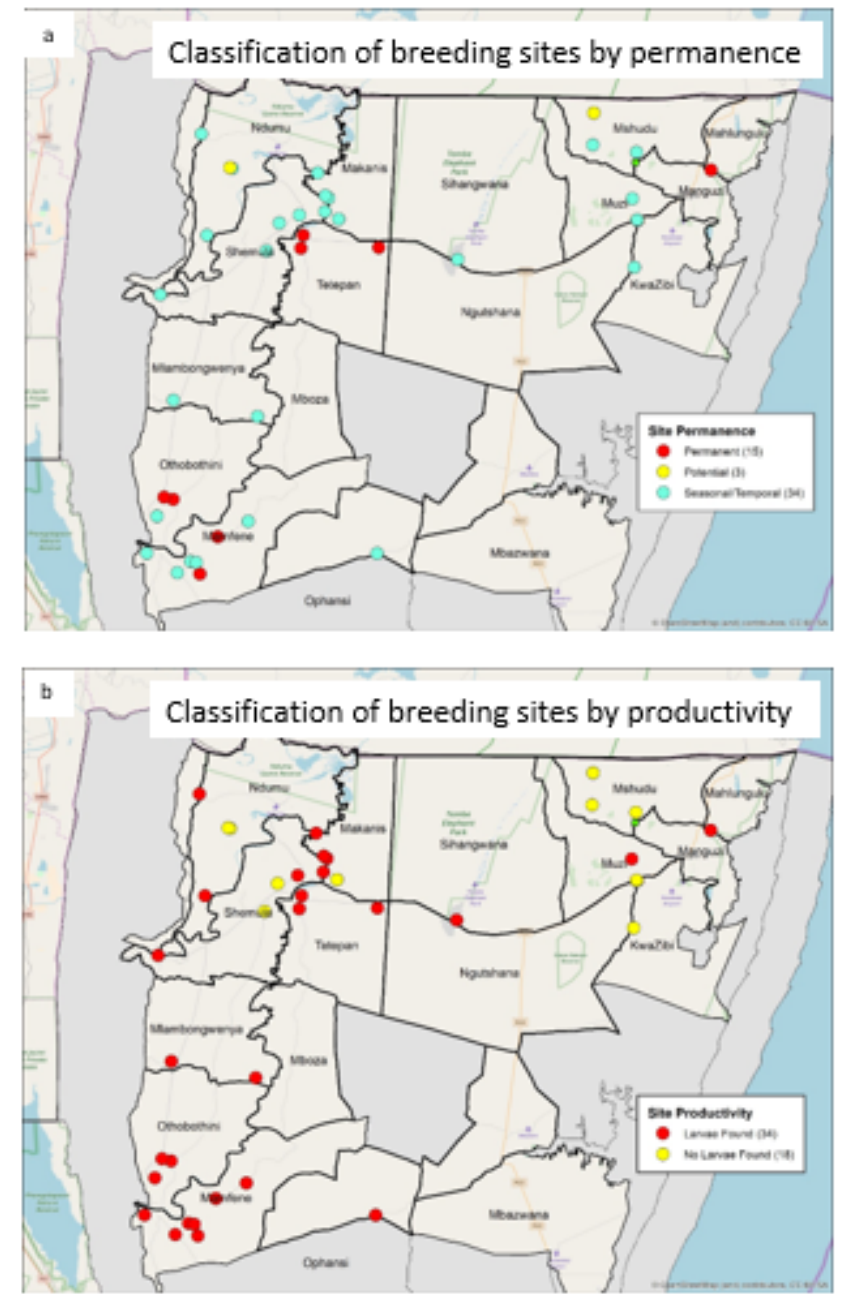

\section{Figure 4}

Breeding sites identified during the prevalence survey classified by a) site permanence and b) productivity.

\section{Supplementary Files}

This is a list of supplementary files associated with this preprint. Click to download.

- SupplementaryTableMalaraJ27Mar2020.docx 